\title{
Non-Cystic Fibrosis Bronchiectasis: from Programmatic Management to Personalized Medicine
}

\author{
Oxana Munteanu ${ }^{a}$ Helmut J.F. Salzer ${ }^{b, c}$ \\ aDepartment of Pneumology and Allergology, State University of Medicine and Pharmacy "Nicolae Testemitanu", \\ Chisinau, Moldova; ${ }^{\mathrm{b}}$ Division of Clinical Infectious Diseases, Research Center Borstel, and ${ }^{\mathrm{C}}$ German Center for \\ Infection Research, Clinical Tuberculosis Center, Borstel, Germany
}

There is a renewed interest in non-cystic fibrosis bronchiectasis (NCFB), which led to increased research activities including the establishment of large NCFB registries such as the European Bronchiectasis Registry (EMBARC) and the United States Bronchiectasis Research Registry (BRR) [1,2]. The growing evidence indicates that NCFB is characterized by an extreme heterogeneity with a wide range of etiologies with various clinical, microbiological, functional, and radiological features. Patients often experience a significant morbidity and are usually difficult to manage with highly variable treatment outcomes [3]. Information on patient characteristics seems to be crucial to provide a personalized treatment approach to improve the management of patients suffering from this heterogenic condition. In this issue of Respiration, McDonnell et al. [4] evaluated the frequency and clinical characteristics of NCFB patients with middlelobe predominant bronchiectasis affecting the right middle-lobe and/or lingula (RMLP). Out of 81 patients, 20 (24.7\%) had RMLP, characterized by a younger age, female predominance, and a lower body mass index compared to patients with the classical bronchiectasis phenotype. Patients with a RMLP phenotype also had a higher incidence of nontuberculous mycobacterial (NTM) infection at diagnosis and experienced a milder disease se-

\section{KARGER}

(ㅇ 2017 S. Karger AG, Basel

E-Mail karger@karger.com

www.karger.com/res verity expressed by fewer symptoms of cough and daily sputum, a lower exacerbation frequency, a higher baseline forced expiratory pressure in $1 \mathrm{~s}$ percentage, and a lower modified bronchiectasis severity index and modified FACED severity score.

Radiological features of NTM pulmonary disease are commonly classified into fibrocavitary or nodular-bronchiectatic disease [5]. The latter is not specific to NTM infections, but the presence of bronchiectasis has been suggested to be typical in patients with Myobacterium avium complex infection. Generally, the clinical course of nodular-bronchiectatic disease seems less aggressive than that of fibrocavitary disease in NTM pulmonary disease [6]. The particularity of the CT image of the RMPL phenotype should always direct the attention to NTM disease as an important differential diagnosis.

Different methods have been proposed to identify discrete groups of NCFB patients. For example, patients can be classified based on clinical phenotypes. A larger study of 1,145 patients identified 4 clusters that revealed significant differences in the quality of life, exacerbations, hospitalizations, and mortality. Clinical features included demographics, comorbidities, and clinical, radiological, functional, and microbiological variables. Patients were grouped in 4 different clusters including (1) "pseudomo-

Dr. Oxana Munteanu

Department of Pneumology and Allergology

State University of Medicine and Pharmacy "Nicolae Testemitanu"

Stefan cel Mare si Sfant Boulevard 165, MD-2004 Chisinau (Moldova)

E-Mail obarbieru@yahoo.com 
nas", (2) "other chronic infection", (3) "daily sputum", and (4) "dry bronchiectasis" [7]. Furthermore, different risk stratification tools have been proposed to predict NCFB patients at risk for mortality, hospitalization, and exacerbation, such as the bronchiectasis severity index or the FACED score $[8,9]$.

The vast majority of NCFB patients can be managed with a conservative treatment strategy, surgical treatment being reserved for focal disease aiming to remove permanently damaged areas of the lung characterized by poor antimicrobial drug penetration that serves as microbiological reservoir leading to recurrent infections. There are limited long-term outcome data for NCFB patients after surgery [10]. NCFB patients with a RMPL phenotype may benefit from surgical treatment, but systematic data are limited.
A major difference of patients with NCFB compared to others is the extreme heterogeneity and chronic course of the disease with recurrent exacerbations and diverse complications with a substantial impact on health-care systems. As the disease itself, the management of patients with NCFB has to be "heterogenic" too. Precise assessment, with a particular focus on the different NCFB patient characteristics, could help to overcome current challenges and to assign the right treatment to the right patient. It needs further research commitment to increase the understanding of this condition to advance patient care from programmatic management to personalized medicine [11].

\section{References}

1 Aksamit TR, O'Donnell AE, Barker A, Olivier KN, Winthrop KL, Daniels ML, et al: Adult bronchiectasis patients: a first look at the United States Bronchiectasis Research Registry. Chest 2016;pii: SDD12-3692(16)623541.

2 Chalmers JD, Aliberti S, Polverino E, Vendrell M, Crichton M, Loebinger M, et al: The EMBARC European Bronchiectasis Registry: protocol for an international observational study. ERJ Open Res 2016;2:pii: 0081-2015. eCollection 2016.

3 Chalmers JD, Aliberti S, Blasi F: Management of bronchiectasis in adults. Eur Respir J 2015; 45:1446-1462.

4 McDonnell MJ, Ahmed M, Das J, O'Mahony M, Breen D, O'Regan A, Gilmartin JJ, Bruzzi J, Rutherford RM: Patterns of disease in patients with middle-lobe predominant bronchiectasis. Respiration 2017;93:406-414.
5 Griffith DE, Aksamit T, Brown-Elliott BA, Catanzaro A, Daley C, Gordin F, et al: An official ATS/IDSA statement: diagnosis, treatment, and prevention of nontuberculous mycobacterial diseases. Am J Respir Crit Care Med 2007;175:367-416.

6 Wassilew N, Hoffmann H, Andrejak C, Lange C: Pulmonary disease caused by non-tuberculous mycobacteria. Respiration 2016;91:386402.

7 Aliberti S, Lonni S, Dore S, McDonnell MJ, Goeminne PC, Dimakou K, et al: Clinical phenotypes in adult patients with bronchiectasis. Eur Respir J 2016;47:1113-1122.

8 Martinez-Garcia MA, de Gracia J, Vendrell Relat M, Giron RM, Maiz Carro L, de la Rosa Carrillo D, et al: Multidimensional approach to non-cystic fibrosis bronchiectasis: the FACED score. Eur Respir J 2014;43:13571367.
9 Chalmers JD, Goeminne P, Aliberti S, McDonnell MJ, Lonni S, Davidson J, et al: The bronchiectasis severity index. An international derivation and validation study. Am J Respir Crit Care Med 2014;189:576-585.

10 Szymanski EP, Leung JM, Fowler CJ, Haney C, Hsu AP, Chen F, et al: Pulmonary nontuberculous mycobacterial infection. A multisystem, multigenic disease. Am J Respir Crit Care Med 2015;192:618-628.

11 Salzer HJ, Wassilew N, Kohler N, Olaru ID, Gunther G, Herzmann C, et al: Personalized medicine for chronic respiratory infectious diseases: tuberculosis, nontuberculous mycobacterial pulmonary diseases, and chronic pulmonary aspergillosis. Respiration 2016;92: 199-214.
NCFB: from Programmatic Management to Personalized Medicine
Respiration 2017;93:404-405 